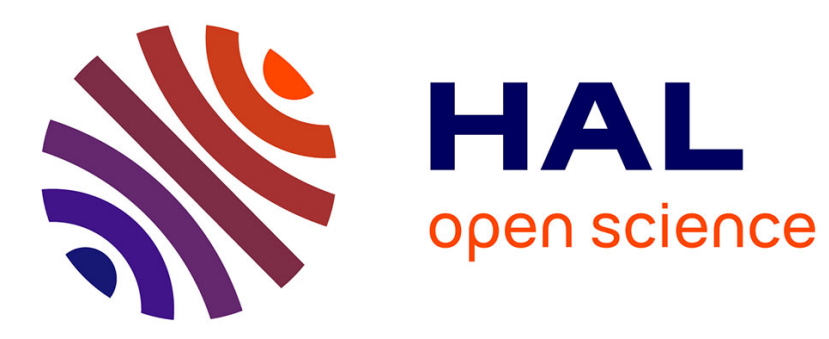

\title{
Blind Minutiae Selection for Standard Minutiae Templates
}

Z Yao, B Vibert, Christophe Charrier, Christophe Rosenberger

\section{To cite this version:}

Z Yao, B Vibert, Christophe Charrier, Christophe Rosenberger. Blind Minutiae Selection for Standard Minutiae Templates. IEEE International Conference on Identity, Security and Behaviour Analysis, Mar 2015, Hong-Kong, China. hal-01150257

\section{HAL Id: hal-01150257 https://hal.science/hal-01150257}

Submitted on 9 May 2015

HAL is a multi-disciplinary open access archive for the deposit and dissemination of scientific research documents, whether they are published or not. The documents may come from teaching and research institutions in France or abroad, or from public or private research centers.
L'archive ouverte pluridisciplinaire HAL, est destinée au dépôt et à la diffusion de documents scientifiques de niveau recherche, publiés ou non, émanant des établissements d'enseignement et de recherche français ou étrangers, des laboratoires publics ou privés. 


\title{
Blind Minutiae Selection for Standard Minutiae Templates
}

\author{
Z. Yao, B. Vibert, C. Charrier, C. Rosenberger \\ Universite de Caen Basse Normandie; ENSICAEN; GREYC UMR 6072, CNRS \\ 17 Rue Claude Bloch, 14000 Caen, France
}

\begin{abstract}
The embedded applications of fingerprint proposed so far are chiefly based on the minutiae template. This kind of system is not resource-free and minutiae template is generally sacrificed to cover the shortage. This paper presents several simple yet efficient no-image minutiae selection approaches (NIMS) for the standard minutiae templates (ISO/IEC 19794-2). With the reduced-templates obtained by using the proposed methods, the overall performance can be guaranteed in comparing with the results generated by the original templates. The interoperability tests are performed with several FVC databases. An additional analysis with the quality of the enrollment samples is also carried out. The experimental results demonstrate the validity and efficiency of the proposed approaches.
\end{abstract}

\section{Introduction}

Due to the advantages in privacy and efficiency requirements, minutiae template-based matching is the dominant technique among the authentication approaches of fingerprint image [8]. The application of minutiae-based matching mainly involves two categories: resource-free systems and embedded employments such as smart-card [1]. The former has almost no limitation of the computing cost and the storage requirement. However, these factors are the prerequisites for the embedded applications, especially for those match-on-card (MOC) systems [10]. In this case, to satisfy such requirements, some on-card applications support only a minutiae template that contains a certain amount of minutiae such as 60 points. Generally, the amount of minutia points of one template could be less than 130 and it is fully dependent on the extractor and the image quality. Therefore, it is necessary to perform a removal or selection of minutiae from the original template before the templates can be used by those resource-limited applications.

A minutiae template is commonly stored as the records of the minutia points consisting of their location and orien- tation information with respect to a 2D plane of the corresponding fingerprint image. In addition to these two types of information, some other characteristics or measurements might also be provided in the template as the vendors are different. In most cases, implementations would compute an image-based quality value for each minutiae and prune those of low qualities [5]. This paper mainly focuses on the international standard minutiae template for the compact card application (ISO/IEC 19794-2:2005) [3]. In stead of choosing minutiae in terms of the image information, this paper made efforts to achieve a blind selection of minutiae from the original template.

The remaining of the paper is organized as follows: Section 2 presents the selection approaches in details, Section 3 gives the experimental results and some discussions. Section 4 concludes the paper.

\section{Approach Description}

The purpose of minutiae selection is to satisfy the memory limitation of the embedded applications. Meanwhile, such an operation should be able to guarantee the overall performance after a set of minutiae points had been pruned from the original template. The MINEX II [5] presents two cases for pruning minutiae points according to the image information: quality-based and distance-based approach with respect to the image center. Vibert et al. [13] proposed several NIMS approaches to perform blind selection with noncompact templates. The kmeans and truncation proposed in [13] are used as the reference. The former is implemented with the Fuzzy c-means [9] algorithm clustering the minutiae of one template into several groups and the points are pruned in terms of their membership grade with regard to the associated cluster(s). However, this method is easy to undulate due to the c-means algorithm. In this paper, without using any image information, we made several attempts for the compact template in terms of template quality, minutiae orientation and location of the minutiae template.

\subsection{Selection with Template Quality}

Quality based approach involves in two factors, one relies on an index of minutiae template quality (could be 
viewed as a no-image quality of fingerprint) [16] and another is derived from a matching approach [2]. For the first one, the quality index based on the convex hull and Delaunay triangulation is applied onto the minutiae template. By using this method, some bad triangles determined by the quality index are figured out. The selection criterion then locked on the vertices of those bad triangles and remove some of them from the original template if they are located on the border of the convex hull. The template could be further reduced by using another factor which is given by equation 1

$Q_{i}=f_{1}\left(d_{i}, D_{T_{1}}, D_{T_{2}}\right)= \begin{cases}0 & \text { if } d_{i}<D_{T_{1}}, \\ 1 & \text { if } d_{i}>D_{T_{2}}, \\ \frac{d_{i}-D_{T_{1}}}{D_{T_{2}}-D_{T_{1}}} & \text { otherwise. }\end{cases}$

Equation 1 is a quality estimation of each minutia point according to the average distance between the minutia itself and the three nearest neighbor points. In the equation, $d_{i}$ is the average distance, $D_{T_{1}}$ and $D_{T_{2}}$ are two empirical threshold which were set to the mean value and a plus with the standard deviation of the minimum distance (to neighbor) of all the minutiae. In the experiment, the mean value is 17 and the standard deviation is 9 . The second factor gives each minutiae an estimated quality value so that it can be used independently for reducing minutiae points. The approach with a combination of these two factors is denoted as 'DVQ' in the experiment. The second factor is named as 'Separation' when it is used independently.

\subsection{Orientation Index}

The regularity of ridge-valley pattern determines their flow direction and the orientation of minutiae points either. In this case, the orientation of minutiae in the range of $[0$, $180]$ and [180, 360] are regarded as the same direction of the ridge-valley pattern. The ridge-valley pattern of a fingerprint could be divided into several orientation areas, as illustrated in figure 1 . Therefore, one can conduct

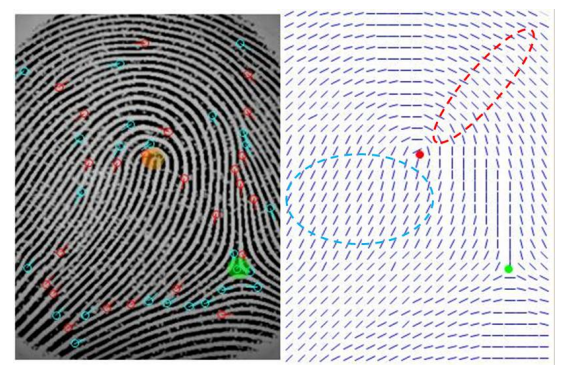

Figure 1: An example of minutiae and orientation field of a fingerprint.

that the orientation of minutiae could be used for such a division. With this consideration, the orientation of each minutia is firstly transferred to the range of [0, 360], and they are further converted into $[0,180]$. Second, minutiae amount is counted for each degree of $[0,180]$ and this measure is sorted in an ascending order. At last, minutiae fell into the small bins are removed according to the desired number of the selection.

Another method based on the orientation of minutia is to divide the angle range of $[0,360]$ into 8 sectors. Next, according to the orientation value of the minutiae, the minutiae number in each sector are counted up and the points are also removed according to the ascending order of the minutiae number of each sector. In the experiment, these two factors are denoted as 'AngHist' and 'AngSec', respectively.

\subsection{Distance Index}

Distance between each minutia and the centroid of the convex hull (polygon) of the minutiae template is also calculate as a potential index for selection. In this study, this kind of distance is used in 3 different ways. First, the distance is simply calculated between each minutia and the centroid of the polygon. We use the centroid of the polygon simply because the quality of an image is unknown and it is not appropriate to use the image center for some samples with only light translation of the foreground (even if the quality is not bad), especially when the image dimension is relatively large (Cf. figure 2 ). In figure 2 , one can observe

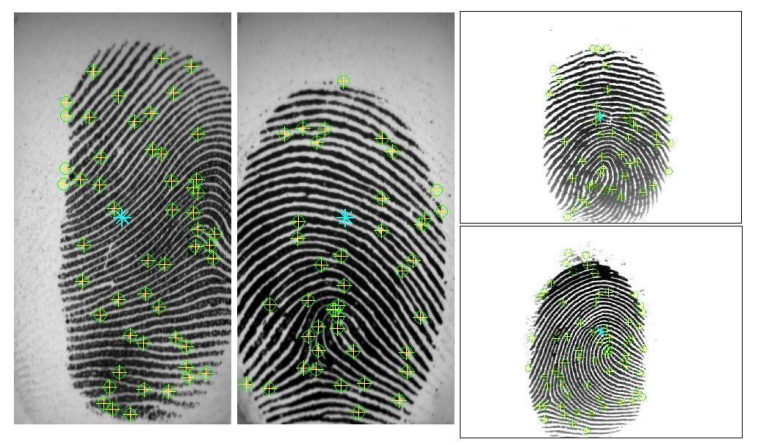

Figure 2: Illustration of the disadvantage of using image center.

that some minutiae are relatively far from the image center (marked by blue star) and removing these minutiae can lead to low genuine matching if the translation of another template is tiny or relatively smaller. For this measurement, the points are pruned according to the largest value of the distances one by one.

Secondly, the distance is obtained for the vertex points of the convex hull only. They are also deleted in terms of the largest value, but the selection might be completed 
with several iterations and each iteration is performed by using the previously reduced template so that the centroid is dynamic. Furthermore, to make a comparison with the floating centroid, a fixed centroid of the convex hull obtained from the original template has been kept during the selection of each iteration. These three indexes are denoted as 'Dist', 'Vertex' and 'VertexFixC' in the experiment.

These approaches could be either combined or performed independently in the experiment. For instance, a combination of the first factor of 'DVQ' and 'Dist' is tested in the experiment (denoted by 'Dela et Dist'). Minutiae type is not considered in this study due to the interoperability analysis.

\section{Experimental Result}

In order to observer the performance of the selection indexes for various datasets, the experiments have been conducted on several trial databases from the Fingerprint Verification Competition (FVC) [7], details are given below.

\subsection{Protocol}

In the experiment, one dataset of FVC2002 and four FVC2004 datasets are involved in a comparative study. Each of the datasets include 100 individuals and 8 samples per individual, 800 images in total. The details of each dataset is given in table 1 .

Table 1: Dataset specification.

\begin{tabular}{|l|c|c|c|}
\hline DB & Sensor & Dim. & Resolution \\
\hline 02DB2A & Optical & $296 \times 560$ & 569dpi \\
\hline 04DB1A & Optical & $640 \times 480$ & 500dpi \\
\hline 04DB2A & Optical & $328 \times 364$ & 500dpi \\
\hline 04DB3A & Thermal & $300 \times 480$ & 512dpi \\
\hline 04DB4A & SFinGe & $288 \times 284$ & about 500dpi \\
\hline
\end{tabular}

As it is given in table 1, the image size of each dataset is different from one another and the resolution is over 500dpi. A glance of the datasets are given by several samples in figure 3. In the experiment, the ISO/IEC standard compact minutiae template is extracted with a commercial fingerprint SDK. With this SDK, we implemented a minutiae extractor and a matching application which is able to perform matching between two images or two templates (with ISO/IEC 19794-2:2005 compact standard) and return an integral number up to 65535 representing the largest matching score. The NIST software Bozorth3 [6] is another matcher involved in the experiment for the interoperability analysis. The ISO/IEC 19794-2 standard recommends the maximal number of minutiae for enrollment and verification is 60 [4]. In this study, the size of the reduced template began with 30 and increased by 2 until 60 . The reduced templates in each

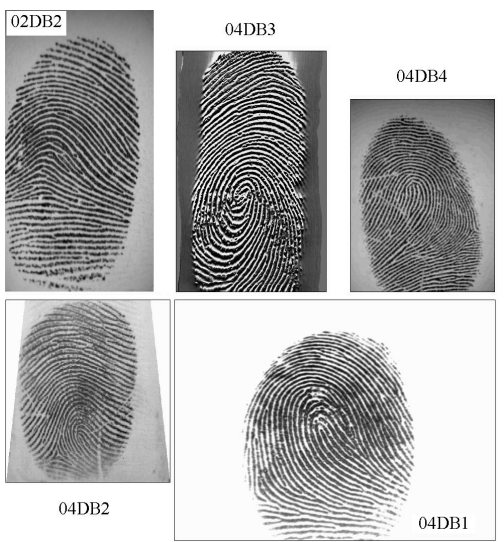

Figure 3: Illustration of dataset samples.

size are generated from the original compact template with all the selection approaches. This is followed by a computation of intra-class and inter-class matching scores, given by a set of genuine matching scores (GMS)

$$
g m s_{i, j, k}=R\left(S_{i, j}, S_{i, k}\right) j \neq k
$$

and $N-1 \times M-1$ impostor matching scores (IMS)

$$
i m s_{i, j, l, k}=R\left(S_{i, j}, S_{l, k}\right) i \neq l \text { and } j \neq k,
$$

where $R$ represents the matcher, $S_{\text {., }}$ denotes the sample, $M$ is the individual number and $N$ is the sample number. In the experiment, the overall performance is represented by a global EER value (or AUC) obtained by assigning the first sample of each individual as the enrollment. The global EER (or AUC) is an average value of 1000 EER (or AUC) values calculated by a random selection (1000 iterations) of 500 samples from the inter-class and 500 samples from the intra-class scores. With this measurement, one can observe how well a selection approach could achieve in comparing with the original template which is denoted as no-selection in the experiment. Nevertheless, the NFIQ [12] is also involved in the this study for observing the effect of quality.

\subsection{Interoperability Analysis}

Grother et al. [4] presented three-way interoperability involving cross matching with multi-vendor templates or matchers. In this study, due to the limitation of available matchers, bozorth 3 is employed for such an analysis. It uses only the location $(x, y)$ and the orientation $o$ of minutiae points of the ANSI/NIST standard template [11,6]. The orientation unit for this type of template is 2 degree, while the unit of the template generated by the SDK is over 5 degree. In the experiment, no transformation is applied onto the minutiae record prior to matching operations.

Theoretically, the FNMR might be higher and the FMR could be lower when the minutiae number of the involved 
templates become larger. However, this is dependent on the matching program, especially with regard to the interoperate matching. With appropriate selection approaches, the overall performance is expected to be increased as the minutiae number reached at a certain amount. In this case, we count the minutiae number of each database, illustrated by boxplots given in figure 4 . In addition, the mean values

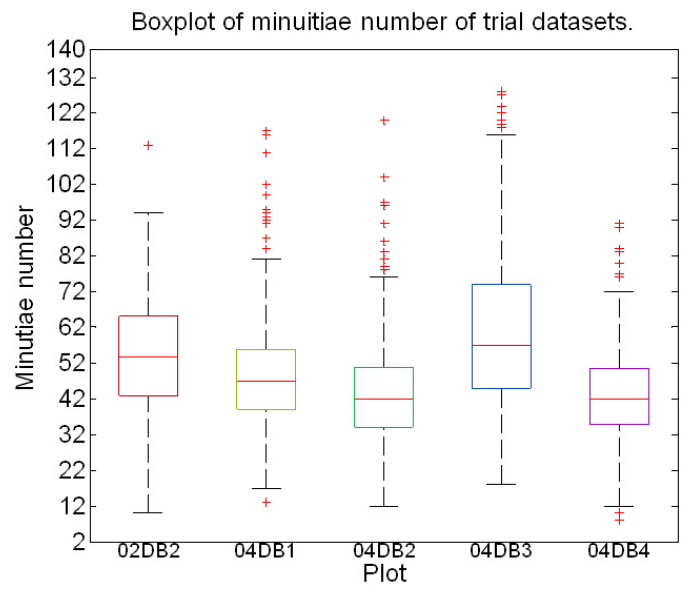

Figure 4: Minutiae number statistics of the trial datasets.

of them are 54, 48, 43, 62 and 43, respectively.

Figure 5 illustrates the evaluation results based on the matching score of Bozorth3 of the reduced template, representing by the global EER values. With the graph results, one can note that the result obtained on FVC2002DB2 is relatively irregular. The overall performance of the reduced template at each number is better than that of the original template. This is attributed to several reasons: 1) the interoperability of matching algorithm, 2) the outlier of minutiae numbers of the dataset and 3) the quality distribution of the dataset. In FVC2002DB2, according to the boxplot in figure 4 , the outlier is quite small and there are not so many large size templates, while the quality distribution mainly concentrates on the first two levels as they are given in figure 4 and table 2, respectively. Particularly, samples whose quality is better than level 3 in each column of this dataset almost accounts for only $5 \%$ except the seventh column.

The other datasets have more outliers, especially FVC2004DB3A from which the worst result is obtained. This dataset has a relatively long whiskers leading to the more error rates which are more distinctive in the left of the $\mathrm{x}$-axis (small size template). However, the increment of the overall performance can be observed from the four datasets of FVC2004. This is further demonstrated by the results obtained by using the homologous matching algorithm (SDK), graphical results are given in figure 6.

The results given in figure 6 are basically consistent with the ones in figure 5. The selection approaches obtained a result on FVC2002DB2A which is almost perfect for this kind of image, and a relatively bad result on FVC2004DB3A. Among all the approaches, the distance index and vertexbased approaches demonstrate a generality when dealing with datasets where the outlier is not very distinctive. They can obtain an acceptable result when the template size is over 50 except FVC2004DB3A. At 50, the second factor of quality-based indexes performs better than the others on FVC2004DB3A due to the existence of a large number of dense template in this dataset. Obviously, the NIMS approach is affected by the number of minutiae as the experiment result demonstrated. This is also a factor of the sample quality, and it is more distinct for a dataset where the sample diversity is relatively noticeable.

\subsection{Effect of Enrollment Quality}

According to the inteoperability analysis, one can observe that both the minutiae number and quality significantly affect the performance of the NIMS approach. However, in order to reveal the significance of the quality of samples to the NIMS approach, this section makes an objective study about the effect of enrollment sample's quality. Yao et al. [14] proposed the impact of enrollment quality on the overall performance when dealing with a trail database. In the experiment, we firstly calculate the quality distribution of the trial datasets, given in table 2 . As it is presented in

Table 2: Quality counting up of the trial databases.

\begin{tabular}{|l|c|c|c|c|c|c|}
\hline DB & L1 & L2 & L3 & L4 & L5 & Under L3 \\
\hline 02DB2 & 361 & 370 & 64 & 5 & 0 & $8.62 \%$ \\
\hline 04DB1 & 512 & 208 & 70 & 4 & 6 & $10 \%$ \\
\hline 04DB2 & 111 & 345 & 277 & 16 & 51 & $43 \%$ \\
\hline 04DB3 & 449 & 214 & 84 & 47 & 6 & $17.13 \%$ \\
\hline 04DB4 & 300 & 324 & 162 & 1 & 13 & $22.00 \%$ \\
\hline
\end{tabular}

the table, samples number for each quality level of NFIQ is figured out and we also calculate a percentage of samples whose qualities are less than and equal to the medium level. This statistic was introduced in the last section but it is not an objective and explicit analysis. In this part, we firstly use three quality metrics, NFIQ, QMF and MQF [15, 16], to generate the quality value of each sample and perform the enrollment selection to the reduced template. However, it is unavoidable that the quality metrics would generate some outliers. In this case, we next calculate the EER value of each sample for the datasets. The enrollment selection is also applied onto the reduced template in terms of this measurement. Specifically, we choose the enrollment (template) among the reduced templates of each individual according to the quality or EER value of the sample.

We simply choose the selection approach that performs relatively well, such as 'Vertex' or 'Separation'. This kind 
of calculation has to be performed for each size of the reduced template so that we use only one dataset as the example to illustrate the effect of enrollment quality, see figure 7.
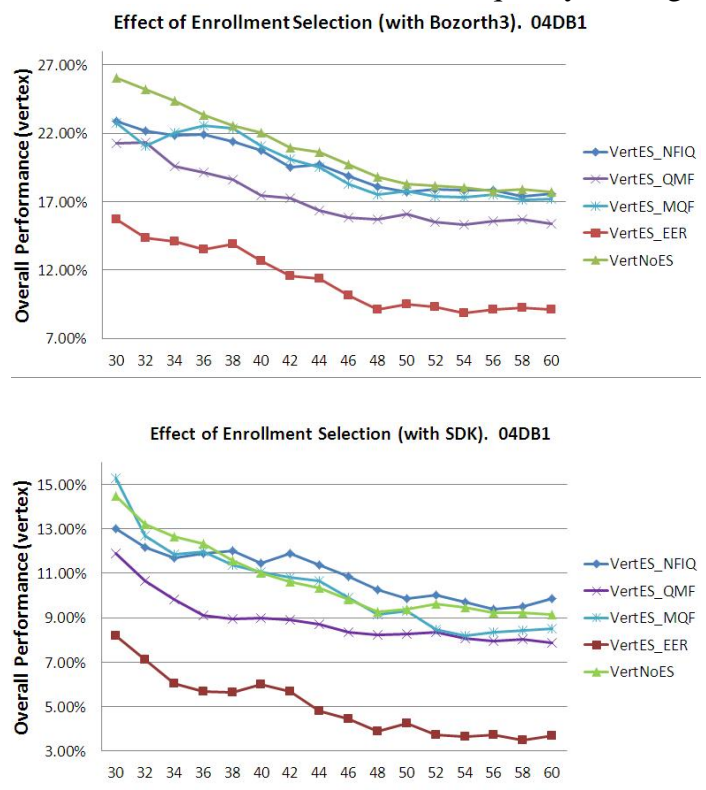

Figure 7: Evaluation results with Bozorth3.

Fig. 7 shows plots of the overall performance, after the enrollment selection had been performed, of the reduced template obtained by 'Vertex' of FVC2004DB1 (we don't use 02DB2 due to the SDK-based global EER of this dataset is almost zero). In the figure, VertES_EER represents the ES result obtained from the reduced templates by using the EER value of the original sample, VertNoES denotes the error rates obtained from the reduced template with no ES and other three correspond to the ES results based on quality metrics. The confidence interval of each error rate in the plot is not provided due to space limitation. Note that two clearly separated points at the same desired number can indicate the distinctive difference between them [16] and the error of the global EER is within $0.5 \%$ by observing 1000 samples. The NFIQ fails to reduce the error rate when the matching score of the SDK is involved. This might be due to the generalization problem for multiple matchers. With the results of QMF and EER, obviously, enrollment quality can affect the NIMS approach.

\section{Conclusion}

In this paper, we present several no-image minutiae selection approaches. In comparing with image-based selection, the NIMS need to be verified in two aspects in general: performance guarantee and effect of quality. The interoperability analysis found that the number of the detected minutiae from the original image is an important factor for the proposed selection criteria. This factor had been considered by many quality assessment approaches by observing the effect of the quality of enrollment samples. Such an enroll- ment selection is also carried out via the interoperate study with variant quality metrics and an objective measure (sample EER could be regarded as the groundtruth). We simply recommend to use 'Vertex' which obtained relatively good results on most of the trial datasets, especially when the span of minutiae number is relatively small. Future studies of this work could focus on the large scale databases and the realistic examinations of the MOC applications.

\section{References}

[1] C. Barral, J.-S. Coron, and D. Naccache. Externalized fingerprint matching. In Biometric Authentication, pages 309-315. Springer, 2004.

[2] K. Cao, E. Liu, L. Pang, et al. Fingerprint matching by incorporating minutiae discriminability. In Biometrics (IJCB), International Joint Conference on, pages 1-6. IEEE, 2011.

[3] O. for Standardization. Iso/iec 19794-2:2005: Information technology biometric data interchange formats part 2: Finger minutiae data, 2005.

[4] P. Grother and W. Salamon. An assessment of iso/iec 7816 card-based 10 match-on-card capabilities 11. 2007.

[5] P. Grother, W. Salamon, C. Watson, et al. Minex iiperformance of match on card algorithms phase ii/iii report. nist interagency report 7477, 2010.

[6] C. I., M. D., E. Tabassi, et al. User's guide to export controlled distribution of nist biometric image software. NIST Report, 2004.

[7] D. Maio, D. Maltoni, R. Cappelli, et al. Fvc2004: Third fingerprint verification competition. In Biometric Authentication, pages 1-7. Springer, 2004.

[8] L. Nanni and A. Lumini. A hybrid wavelet-based fingerprint matcher. Pattern Recognition, 40(11):3146-3151, 2007.

[9] R. Nock and F. Nielsen. On weighting clustering. Pattern Analysis and Machine Intelligence, IEEE Transactions on, 28(8):1223-1235, Aug 2006.

[10] C. T., Y. W., and X. Jiang. On-card matching. In Encyclopedia of Biometrics, pages 1014-1021. Springer, 2009.

[11] E. Tabassi, P. Grother, W. Salamon, et al. Minutiae interoperability. In BIOSIG, pages 13-30, 2009.

[12] E. Tabassi, C. Wilson, and C. Watson. Nist fingerprint image quality. NIST Res. Rep. NISTIR7151, 2004.

[13] B. Vibert, J.-M. Le Bars, C. Charrier, and C. Rosenberger. Comparative study of minutiae selection algorithms for iso fingerprint templates. In Media Watermarking, Security, and Forensics XVI, Proceedings of SPIE, February 2015.

[14] Z. Yao, C. Charrier, and C. Rosenberger. Utility validation of a new fingerprint quality metric. In International Biometric Performance Conference 2014. National Insititure of Standard and Technology (NIST), April 2014.

[15] Z. Yao, J.-M. LeBars, C. Charrier, and C. Rosenberger. Fingerprint quality assessment combining blind image quality, texture and minutiae features. In International Conference on Information Systems Security and Privacy, Feb. 2015.

[16] Z. Yao, J.-M. LeBars, C. Charrier, and C. Rosenberger. Quality assessment of fingerprints with minutiae delaunay triangulation. In International Conference on Information Systems Security and Privacy, Feb. 2015. 


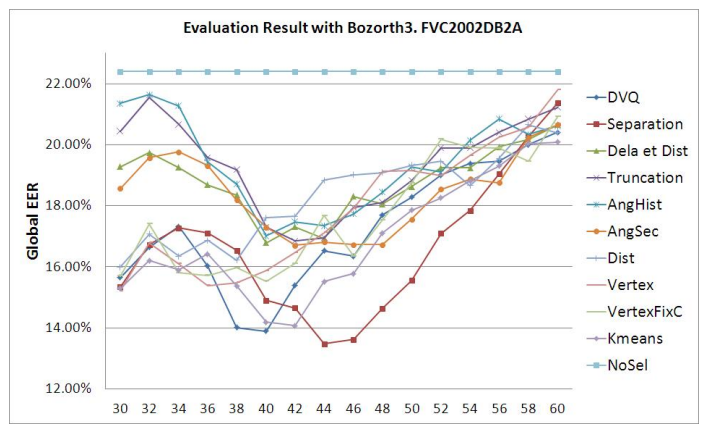

(a) FVC2002DB2A

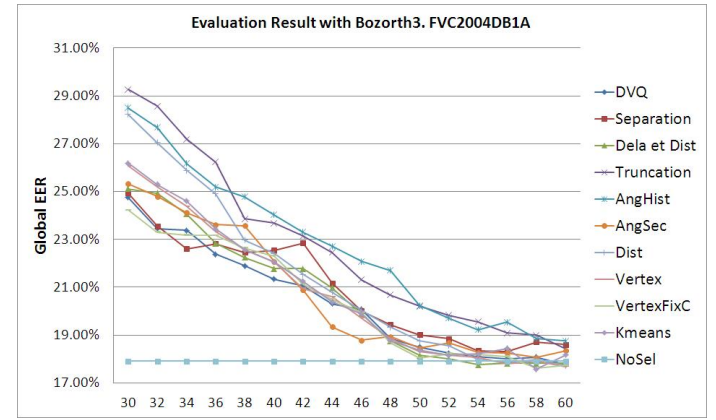

(b) FVC2004DB1A

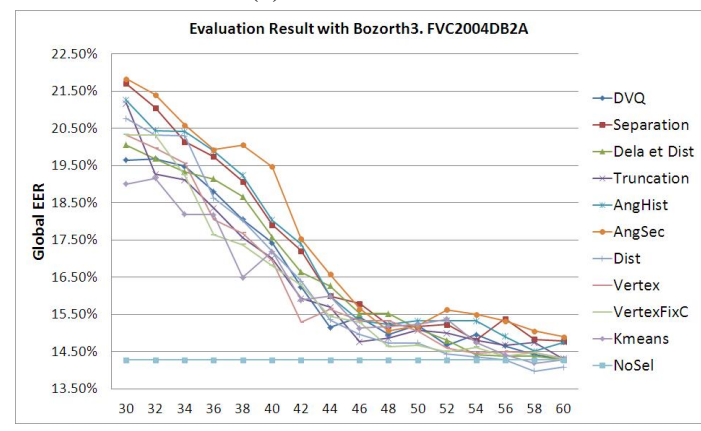

(c) FVC2004DB2A

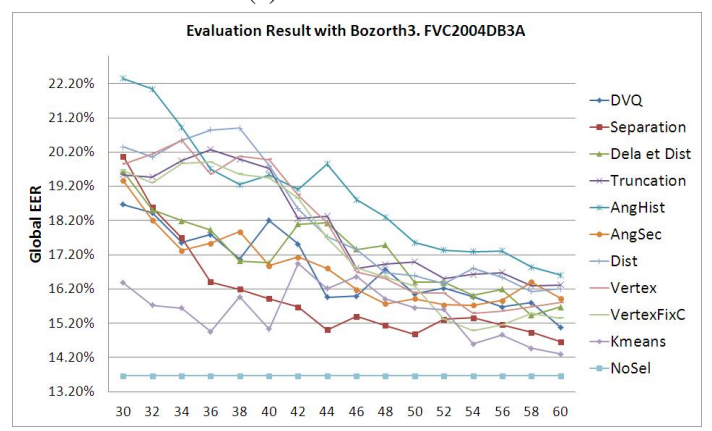

(d) FVC2004DB3A

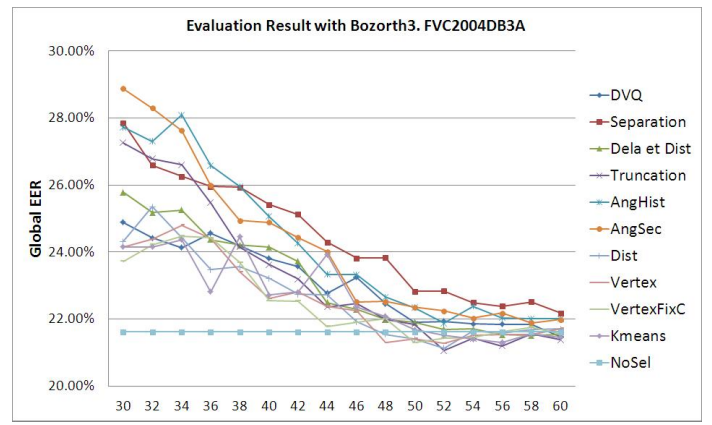

(e) FVC2004DB4A

Figure 5: Evaluation results based on Bozorth3.

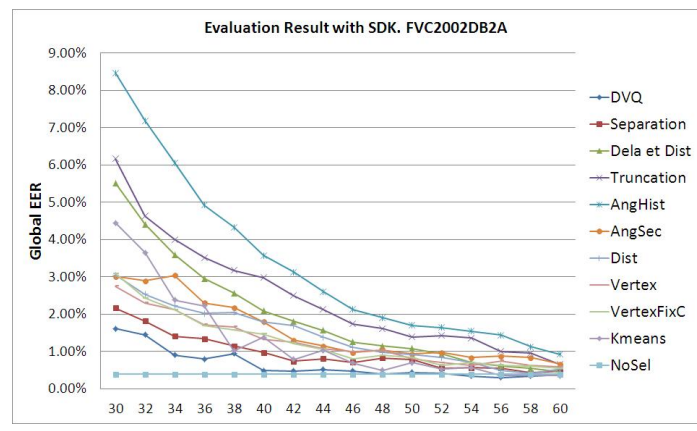

(a) FVC2002DB2A

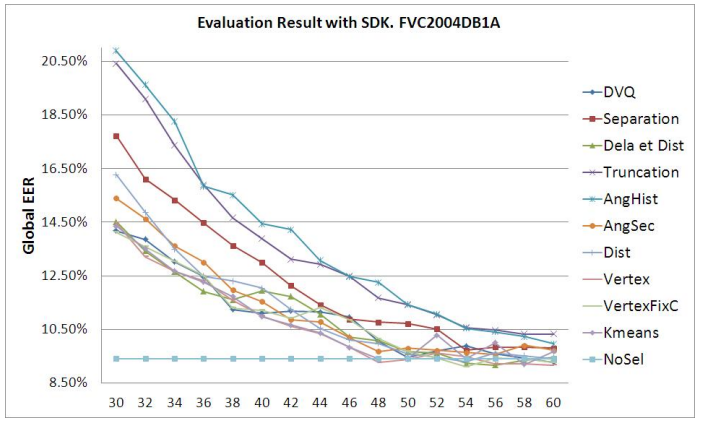

(b) FVC2004DB1A

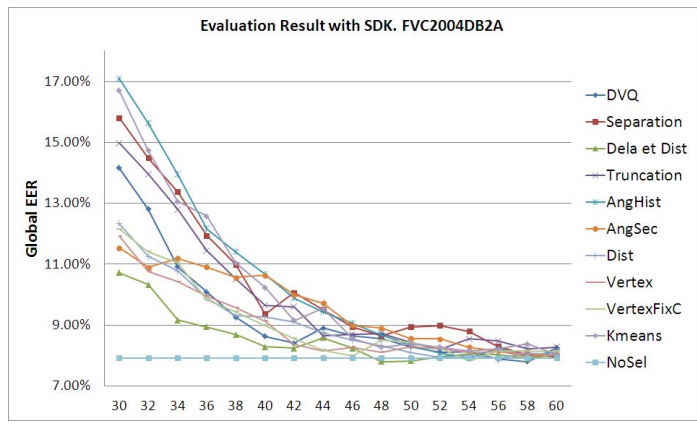

(c) FVC2004DB2A

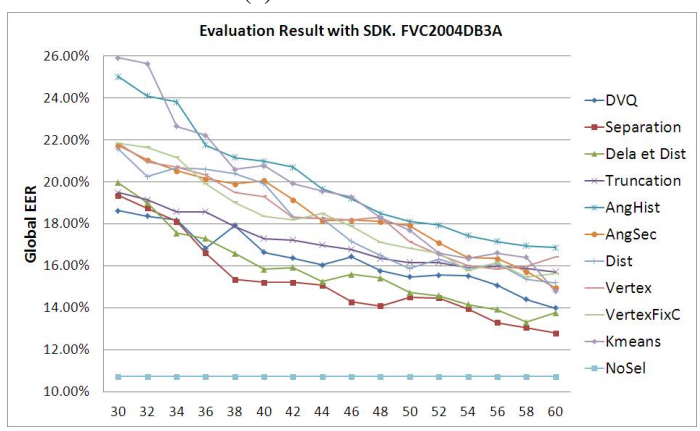

(d) FVC2004DB3A

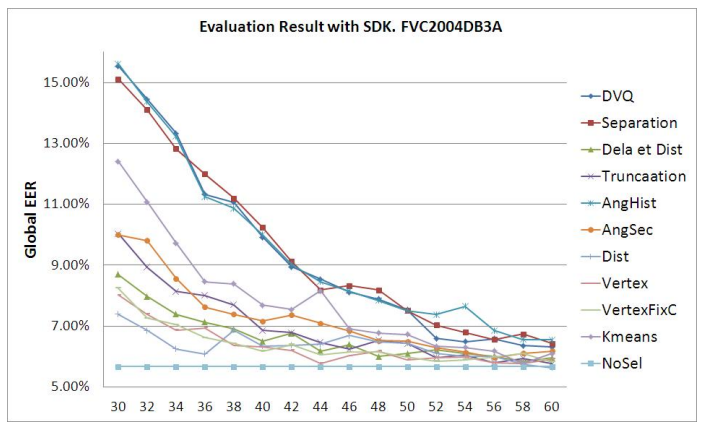

(e) FVC2004DB4A

Figure 6: Evaluation results based on SDK. 